\title{
Association of Menopausal Age with Unruptured Intracranial Aneurysm Morphology
}

\author{
Sushrut Dharmadhikari ${ }^{a} \quad$ Kunakorn Atchaneeyasakul ${ }^{a}$ Sudheer Ambekar ${ }^{b}$ \\ Vasu Saini $^{\mathrm{b}}$ Diogo C. Haussen ${ }^{\mathrm{b}}$ Dileep Yavagal ${ }^{\mathrm{a}} \mathrm{b}$ \\ aDepartment of Neurology, University of Miami - Miller School of Medicine, Miami, FL, USA; \\ ${ }^{b}$ Department of Neurosurgery, University of Miami - Miller School of Medicine, Miami, FL, USA
}

\section{Keywords}

Endovascular therapy $\cdot$ Estrogen $\cdot$ Menopause $\cdot$ Unruptured intracranial aneurysms

\begin{abstract}
Background: The prevalence of unruptured intracranial aneurysms (UIAs) increases rapidly in aging women compared with younger women. The impact of menopausal age on UIAs and treatment outcomes with endovascular therapy has not been well studied. We hypothesized that premenopausal age may have a protective effect on presentation size and treatment outcomes. Objective: To evaluate the association of menopause with UIA size and outcome with endovascular therapy. Methods: Retrospective analysis of consecutive female patients with UIAs treated with endovascular therapy at our academic tertiary care center. UIA characteristics, complications, and outcomes were recorded and compared. Results: 117 patients were included: 23 patients in the premenopausal age (PRM) group and 94 in the postmenopausal age (POM) group. 93.6\% of all aneurysms in the PRM group were in the internal carotid artery (ICA) segments $(p<0.05)$. Hence only ICA segment aneurysms were further studied. A total of 21 patients in the PRM group and 60 in the POM group were found to have ICA segment aneurysms. Baseline characteristics were similar between the 2 groups. The mean size of the aneurysms in the PRM group was $8.6 \pm 3.9$ versus $10.8 \pm 5.6 \mathrm{~mm}$ in the POM group
\end{abstract}

Oral Data-Blitz Presentation at: 67th Annual Meeting of the American Academy of Neurology, April 2015, Washington, DC, USA.

Poster Presentation at: 140th Annual Meeting of the American Neurological Association, September 2015, Chicago, IL, USA.

8th Annual Meeting of the Society of Vascular and Interventional Neurology, October 2015, Bonita Springs, $\mathrm{FL}$, USA. 
( $p=0.055)$. There was a trend to higher aneurysm neck size seen in the POM group $(4.7 \pm 2.5$ vs. $3.7 \pm 1.7 \mathrm{~mm} ; p=0.07)$. The number of aneurysm lobes was higher in the PRM group (1.23 \pm 0.54 vs. $1.07 \pm 0.31 ; p=0.18$ ). In multivariate analysis, the PRM group had a significantly higher number of UIA lobes. Complications and endovascular therapy outcomes were similar between the 2 groups. Conclusions: A trend to increased UIA maximal diameter and neck size was seen in the POM group compared to the PRM group. The PRM group had a significantly higher number of UIA lobes. Larger prospective trials are needed to confirm these findings.

(C) 2019 S. Karger AG, Base

\section{Introduction}

Unruptured intracranial aneurysms (UIAs) occur about twice as often in women as in men [1]. The prevalence of UIAs in women increases more rapidly with age: $2.7 \%$ in women $<40$ years versus $5.4 \%$ in women $>50$ years [1]. A reduction in estrogen levels has been suggested to have a pathogenic role in aneurysm formation [2]. Thus, a physiologic decrease in estrogen with menopause could explain this rapid increase. The impact of menopause on UIAs and their treatment outcomes with endovascular therapy have, however, not been systematically studied.

We hypothesized that premenopausal age may have a protective role on presentation size and treatment outcomes in patients with UIAs compared to postmenopausal age.

\section{Methods}

After obtaining IRB approval, we retrospectively reviewed our database of all consecutive patients undergoing endovascular neurosurgical procedures from April 2008 to April 2014. Female patients harboring UIAs which were treated with endovascular therapy at our institution were included in the study. Male patients and patients with aneurysms associated with other vascular malformations, like anteriovenous malformations, were excluded from the study. The average age of menopause (51 years) in the Western world was used to divide patients into a premenopausal age (PRM) group and a postmenopausal age (POM) group [3].

Basic demographics including age, history of hypertension, diabetes, dyslipidemia, smoking, alcohol abuse, use of hormone replacement therapy, and use of oral contraceptive pills were collected. Aneurysm characteristics included location and number of aneurysms, size of treated aneurysm, number of lobes of treated aneurysm, and aspect ratio of treated aneurysm. Morphological parameters of the aneurysms were acquired from digital subtraction angiography imaging in all patients based on previously described methods $[4,5]$. Modality of treatment utilized in each patient was recorded. Procedural outcomes were determined by degree of occlusion (Raymond-Roy classification). Posttreatment and postprocedural complications were recorded. Follow-up angiographic data, if available, was collected.

Univariate and multivariate analysis of patient with unruptured internal carotid artery (ICA) segment aneurysms treated using endovascular means was separately performed. Data was analyzed in a univariate analysis using the $\chi^{2}$ test with odds ratio and one-way ANOVA using SPSS software (version 22; IBM Corporation, Armonk, NY, USA). Variables with a $p$ value $<0.20$ were included in a multivariate logistic regression model to evaluate the association with POM. Statistical significance was designated as $p$ values $<0.05$.

\section{Results}

A total of 4,889 endovascular neurosurgical procedures were performed at our institution from April 2008 to April 2014. Among these, a total of 117 women were found to be eligible for our study: 23 were in the PRM group (with a mean age of $43.2 \pm 7$ years) and 94 
Table 1. Patient demographics and aneurysm descriptions of all women with unruptured intracerebral aneurysms treated using endovascular means

\begin{tabular}{|c|c|c|c|}
\hline & $\begin{array}{l}\text { Premenopausal } \\
\text { group }(n=23)\end{array}$ & $\begin{array}{l}\text { Postmenopausal } \\
\text { group }(n=94)\end{array}$ & $p$ value \\
\hline Mean age \pm SD, years & $43.2 \pm 7$ & $63.9 \pm 7.5$ & $<0.01^{*}$ \\
\hline Hypertension & $34.7 \%$ & $60.8 \%$ & $0.034^{*}$ \\
\hline Diabetes & $4.3 \%$ & $10.8 \%$ & 0.69 \\
\hline Dyslipidemia & $21.7 \%$ & $34.7 \%$ & 0.23 \\
\hline Smoking $\leq 2$ years & $31.8 \%$ & $17.9 \%$ & 0.23 \\
\hline Alcohol abuse & $0 \%$ & $1.1 \%$ & 0.6 \\
\hline Drug abuse & $0 \%$ & $3.4 \%$ & 0.37 \\
\hline Current birth control & $0 \%$ & $0 \%$ & - \\
\hline \multicolumn{4}{|l|}{ Aneurysm } \\
\hline Size & $9.3 \pm 4.8$ & $10.2 \pm 5.5$ & 0.42 \\
\hline Neck & $4.1 \pm 2.1$ & $4.3 \pm 2.4$ & 0.6 \\
\hline Height & $8.6 \pm 4.4$ & $8.6 \pm 4.9$ & 0.98 \\
\hline Parent vessel diameter & $3 \pm 0.6$ & $2.9 \pm 0.7$ & 0.86 \\
\hline Aspect ratio & $2.17 \pm 0.74$ & $2.24 \pm 1.16$ & 0.71 \\
\hline Size ratio & $3.29 \pm 1.96$ & $3.6 \pm 2.11$ & 0.51 \\
\hline Aneurysms & & & 0.98 \\
\hline 1 & 13 & 65 & \\
\hline 2 & 9 & 18 & \\
\hline 3 & 1 & 9 & \\
\hline Mean & $1.48 \pm 0.59$ & $1.43 \pm 0.82$ & 0.77 \\
\hline Location of treated aneurysm & & & $0.03^{*}$ \\
\hline ACommA & 0 & 8 & \\
\hline PCommA & 0 & 11 & \\
\hline MCA & 0 & 0 & \\
\hline $\mathrm{BA}$ & 1 & 7 & \\
\hline PICA & 0 & 1 & \\
\hline ICA branches & 21 & 60 & \\
\hline Other & 1 & 7 & \\
\hline \multicolumn{4}{|l|}{ Side of treated aneurysm } \\
\hline Right & $45 \%$ & $50 \%$ & \\
\hline Left & $55 \%$ & $50 \%$ & \\
\hline Lobes in treated aneurysm & & & 0.09 \\
\hline 1 & 19 & 90 & \\
\hline 2 & 3 & 3 & \\
\hline 3 & 1 & 1 & \\
\hline Mean & $1.21 \pm 0.51$ & $1.05 \pm 0.26$ & 0.15 \\
\hline Raymond-Roy classification & & & 0.81 \\
\hline 1 & 15 & 64 & \\
\hline 2 & 4 & 20 & \\
\hline 3 & 4 & 10 & \\
\hline Complications of treatment & $17.4 \%$ & $19.6 \%$ & 1 \\
\hline Angiographic follow-up & $100 \%$ & $75.5 \%$ & $0.02^{*}$ \\
\hline
\end{tabular}

ACommA, anterior communicating artery; PCommA, posterior communicating artery; MCA, middle cerebral artery; BA, basilar artery; PICA, posterior inferior cerebellar artery; ICA, internal carotid artery.

* Statistically significant.

were in the POM group (with a mean age of $63.9 \pm 7.5$ years). Among the baseline characteristics, hypertension was present in $61 \%$ of all patients in the POM group versus $35 \%$ in the PRM group $(p=0.034)$. All other baseline characteristics including diabetes, dyslipidemia, smoking and alcohol consumption, and hormone replacement therapy were found to be similar between these 2 groups (Table 1 ). Aneurysms were analyzed by location, and patients 
Table 2. Univariate and multivariate analysis of patient and aneurysm characteristics of women with unruptured internal carotid artery segment aneurysms treated using endovascular means

\begin{tabular}{|c|c|c|c|c|}
\hline & $\begin{array}{l}\text { Premenopausal } \\
\text { group }(n=21)\end{array}$ & $\begin{array}{l}\text { Postmenopausal } \\
\text { group }(n=60)\end{array}$ & $\begin{array}{l}\text { Univariate } \\
p \text { value }\end{array}$ & $\begin{array}{l}\text { Multivariate } \\
p \text { value }\end{array}$ \\
\hline Mean age $\pm S D$, years & $42.6 \pm 7.1$ & $63.5 \pm 7.9$ & $<0.01^{*}$ & \\
\hline Hypertension & $38.1 \%$ & $56.67 \%$ & 0.2 & \\
\hline Diabetes & $4.7 \%$ & $10 \%$ & 0.46 & \\
\hline Dyslipidemia & $23.8 \%$ & $26.7 \%$ & 0.79 & \\
\hline Smoking $\leq 2$ years & $28.6 \%$ & $18.3 \%$ & 0.36 & \\
\hline Alcohol abuse & $0 \%$ & $1.7 \%$ & 0.54 & \\
\hline Drug abuse & $0 \%$ & $5 \%$ & 0.28 & \\
\hline Hormone replacement therapy usage & $0 \%$ & $0 \%$ & - & \\
\hline \multicolumn{5}{|l|}{ Aneurysm } \\
\hline Size & $8.6 \pm 3.9$ & $10.8 \pm 5.6$ & 0.055 & 0.081 \\
\hline Neck & $3.7 \pm 1.7$ & $4.7 \pm 2.5$ & 0.071 & 0.567 \\
\hline Height & $8 \pm 3.9$ & $9 \pm 5.2$ & 0.34 & \\
\hline Parent vessel diameter & $3.1 \pm 0.5$ & $3.2 \pm 0.5$ & 0.15 & 0.228 \\
\hline Aspect ratio & $2.17 \pm 0.78$ & $2.17 \pm 1.16$ & 0.98 & \\
\hline Size ratio & $2.87 \pm 1.41$ & $3.3 \pm 1.62$ & 0.2 & \\
\hline Aneurysms & & & 0.43 & \\
\hline 1 & 12 & 45 & & \\
\hline 2 & 8 & 11 & & \\
\hline 3 & 1 & 4 & & \\
\hline Mean & $1.48 \pm 0.60$ & $1.34 \pm 0.66$ & 0.41 & \\
\hline Side of treated aneurysm & & & 0.83 & \\
\hline Right & $42.9 \%$ & $43.3 \%$ & & \\
\hline Left & $57.1 \%$ & $56.7 \%$ & & \\
\hline Lobes & & & $0.08^{*}$ & $0.026^{*}$ \\
\hline 1 & 17 & 57 & & \\
\hline 2 & 3 & 2 & & \\
\hline 3 & 1 & 1 & & \\
\hline Mean & $1.23 \pm 0.54$ & $1.07 \pm 0.31$ & 0.18 & \\
\hline Raymond-Roy classification & & & 0.9 & \\
\hline 1 & 14 & 36 & & \\
\hline 2 & 4 & 12 & & \\
\hline 3 & 3 & 8 & & \\
\hline Complications of treatment & $19 \%$ & $18.33 \%$ & 0.942 & \\
\hline Angiographic follow-up & $100 \%$ & $76.67 \%$ & 0.052 & \\
\hline
\end{tabular}

* Statistically significant.

in the PRM group were found to have a higher percentage of aneurysms in the ICA segments as compared to the POM group ( $p=0.03$ ) and, hence, these patients were selected for further analysis.

ICA segment aneurysms were seen in 81 patients $(76.7 \%$ in the supraclinoid segment and $23.3 \%$ in the cavernous segment). A total of 21 patients in the PRM group and 60 in the POM group were found to have ICA segment aneurysms. All these patients had similar baseline characteristics. Patients in the POM group showed a trend to a greater aneurysm size (10.8 \pm $5.6 \mathrm{~mm})$ as compared to patients in the PRM group $(8.6 \pm 3.9 \mathrm{~mm})$ on univariate analysis $(p=0.055)$. A trend to a greater aneurysm neck size was also seen in the POM group versus the PRM group ( $4.7 \pm 2.5$ vs. $3.7 \pm 1.7 \mathrm{~mm} ; p=0.07$ ). Interestingly, patients in the PRM group were found to have a greater mean number of lobes compared to patients in the POM group $(p=0.08)$. All other aneurysm characteristics were found to be similar between the 2 groups. 
Patients had similar numbers of major and minor complications of treatment and outcomes (Table 2).

In multivariate analysis, statistical significance was found for a higher number of UIA lobes in the PRM group ( $p=0.026)$, while all other variables were found to be statistically nonsignificant.

\section{Discussion}

Intracranial saccular aneurysms are fairly common in the general population with an estimated prevalence of around 1-5\% [6]. Pathologically, they are characterized by localized structural deterioration of the arterial wall with loss of the internal elastic lamina and disruption of the media [7]. Risk factors for cerebral aneurysms include autosomal dominant polycystic kidney disease, a family history of cerebral aneurysms or subarachnoid hemorrhage, female sex, older age, hypertension, and smoking $[1,2,8,9]$. The impact of postmenopausal age, on UIAs and treatment outcomes with endovascular therapy have not been well studied. In this study, we found a trend to increased UIA maximal diameter and neck size in the POM group compared to the PRM group. Although, the PRM group had a significantly higher number of UIA lobes.

In a large meta-analysis by Vlak et al. [1], women were found to suffer twice as often from UIAs as men. In this same study, the prevalence of UIAs increased from $2.7 \%$ in women $<40$ years to $5.4 \%$ in those $>50$ years, suggesting a possible role of lack of estrogen in aneurysm formation [1]. A recent study additionally showed a modestly greater clustering of intracranial aneurysms in women with a family history of aneurysms [10].

In our study, we only evaluated UIAs in women treated with endovascular therapy and, hence, did not evaluate a gender disparity. However, we did have a greater number of women in the POM group than in the PRM group ( $n=94$ vs. 23 ). This finding could be confounded by the increase in age alone, since we used the standard menopausal age of 51 years as cutoff.

Two major epidemiological studies have evaluated rupture risk of intracranial aneurysms: The International Study of Unruptured Intracranial Aneurysms (ISUIA) and the Unruptured Cerebral Aneurysms Study (UCAS). Both these prospective studies have determined aneurysm size to correlate with rupture rates [11, 12]. A study by Hoh et al. [13] showed ruptured aneurysms to have significantly greater dome heights, aspect ratios, maximum diameters, and size ratios than unruptured aneurysms. In our study, we found a trend to increased UIA maximal diameter in the POM group compared to the PRM group $(10.8 \pm 5.6$ vs. $8.6 \pm 3.9 \mathrm{~mm}$ ). These morphological characteristics suggested a greater rupture risk of aneurysms in the POM group as compared to aneurysms in the PRM group based on prior studies. However, endovascular therapy outcome analysis in our study did not show any difference in the procedural outcome (Raymond-Roy classification) or the complication rate between the PRM and the POM groups. To confirm the hypothesis of a higher rupture rate due to the trend to increased UIA diameter in the POM group, larger prospective studies need to be done.

Several studies have supported a hormonal effect on risk of aneurysmal subarachnoid hemorrhage. Harrod et al. [14] concluded that deficiencies in estrogen-mediated cerebral homeostasis in menopausal and postmenopausal women was a potential factor contributing to cerebral aneurysm pathogenesis and rupture. A population-based cohort study found a lower risk for subarachnoid hemorrhage in premenopausal women compared with agematched postmenopausal women, especially in those without a history of smoking or hypertension [15]. In our study, we found the mean ICA aneurysm size to be $8.6 \mathrm{~mm}$ in the PRM group versus $10.8 \mathrm{~mm}$ in the POM group $(p=0.055)$. The mean aneurysm neck size was found 
to be $3.7 \mathrm{~mm}$ in the PRM group versus $4.7 \mathrm{~mm}$ in the POM group ( $p=0.07)$. This finding in POM women may be attributed to a loss of vascular protective effect of estrogen in this population.

However, interestingly, the patients in the PRM group were found to have a greater number of bi-lobed (3/21 vs. $2 / 60)$ and tri-lobed (1/21 vs. $1 / 60)$ aneurysms as compared to the patients in the POM group. Lobulation has been found to weakly correlate with rupture risk in a few studies $[5,16]$. In our study, the PRM group had a significantly higher number of UIA lobes. There are likely 2 possibilities from this scenario: (1) lobulation may be associated with premenopausal age but there is no correlation with rupture risk; (2) lobulation correlates with rupture risk but the association with the PRM group is by chance in our cohort. The clinical significance of this finding is unclear at this time and needs to be further evaluated.

\section{Limitations}

We acknowledge that our study has certain limitations. Given the retrospective nature of the study, we cannot eliminate a potential treatment bias contributing to the findings in the study. Additionally, we do not have a documented "true menopausal status" for the patients in our study and a lack of hormonal measurements. The use of the mean menopausal age to striate groups can potentially be a source of selection bias, and the selection of group by age could be a confounder in our study. Future studies may also include a male control cohort with similar other demographics. Other limitations include the selection of ICA aneurysm cases only.

\section{Conclusion}

The results of this study demonstrate that women of premenopausal age have a higher numbers of aneurysm lobes, while those of postmenopausal age tend to have a larger UIA size. Larger prospective studies focused on the menopausal status and the risk of rupture in this population are required to verify the findings of our study.

\section{Acknowledgements}

We would like to acknowledge the Endovascular Surgical Neuroradiology team, Jackson Memorial Hospital, and the University of Miami, Miami, FL, USA.

\section{Disclosure Statement}

Dileep Yavagal is a consultant for Aldegan/Cytomedix, Inc., EV3 Neurovascular/Covidien, and Stryker Neurovascular. All the other authors have nothing to disclose.

\section{Author Contributions}

Design or conceptualization of the study: S.D., S.A., and D.Y.

Acquisition of data: S.D., K.A., and V.S.

Analysis or interpretation of the data: S.D., K.A., and D.Y.

Drafting or revising the manuscript for intellectual content: all listed authors.

Study supervision: D.Y. 


\begin{tabular}{l|l}
\hline DOI: $10.1159 / 000496701$ & $\begin{array}{l}\text { C } 2019 \text { S. Karger AG, Basel } \\
\text { www.karger.com/ine }\end{array}$ \\
\hline
\end{tabular}

Dharmadhikari et al.: Association of Menopause with Unruptured Intracranial Aneurysm

\section{References}

1 Vlak MH, Algra A, Brandenburg R, Rinkel GJ. Prevalence of unruptured intracranial aneurysms, with emphasis on sex, age, comorbidity, country, and time period: a systematic review and meta-analysis. Lancet Neurol. 2011 Jul;10(7):626-36.

2 Stober T, Sen S, Anstätt T, Freier G, Schimrigk K. Direct evidence of hypertension and the possible role of postmenopause oestrogen deficiency in the pathogenesis of berry aneurysms. J Neurol. 1985;232(2):67-72.

3 Kato I, Toniolo P, Akhmedkhanov A, Koenig KL, Shore R, Zeleniuch-Jacquotte A. Prospective study of factors influencing the onset of natural menopause. J Clin Epidemiol. 1998 Dec;51(12):1271-6.

4 Dhar S, Tremmel M, Mocco J, Kim M, Yamamoto J, Siddiqui AH, et al. Morphology parameters for intracranial aneurysm rupture risk assessment. Neurosurgery. 2008 Aug;63(2):185-96.

5 Beck J, Rohde S, el Beltagy M, Zimmermann M, Berkefeld J, Seifert V, et al. Difference in configuration of ruptured and unruptured intracranial aneurysms determined by biplanar digital subtraction angiography. Acta Neurochir (Wien). 2003 Oct;145(10):861-5.

6 Brown RD Jr, Broderick JP. Unruptured intracranial aneurysms: epidemiology, natural history, management options, and familial screening. Lancet Neurol. 2014 Apr;13(4):393-404.

7 Brisman JL, Song JK, Newell DW. Cerebral aneurysms. N Engl J Med. 2006 Aug;355(9):928-39.

8 Taylor CL, Yuan Z, Selman WR, Ratcheson RA, Rimm AA. Cerebral arterial aneurysm formation and rupture in 20,767 elderly patients: hypertension and other risk factors. J Neurosurg. 1995 Nov;83(5):812-9.

9 Vlak MH, Rinkel GJ, Greebe P, Algra A. Independent risk factors for intracranial aneurysms and their joint effect: a case-control study. Stroke. 2013 Apr;44(4):984-7.

10 Sijtsma LC, Rinkel GJ, Ruigrok YM. Sex-related clustering of intracranial aneurysms within families. Stroke. 2015 Apr;46(4):1107-9.

11 Wiebers DO, Whisnant JP, Huston J 3rd, Meissner I, Brown RD Jr, Piepgras DG, et al.; International Study of Unruptured Intracranial Aneurysms Investigators. Unruptured intracranial aneurysms: natural history, clinical outcome, and risks of surgical and endovascular treatment. Lancet. 2003 Jul;362 (9378):103-10.

12 Morita A, Kirino T, Hashi K, Aoki N, Fukuhara S, Hashimoto N, et al.; UCAS Japan Investigators. The natural course of unruptured cerebral aneurysms in a Japanese cohort. N Engl J Med. 2012 Jun;366(26):2474-82.

13 Hoh BL, Sistrom CL, Firment CS, Fautheree GL, Velat GJ, Whiting JH, et al. Bottleneck factor and height-width ratio: association with ruptured aneurysms in patients with multiple cerebral aneurysms. Neurosurgery. 2007 Oct;61(4):716-22.

14 Harrod CG, Batjer HH, Bendok BR. Deficiencies in estrogen-mediated regulation of cerebrovascular homeostasis may contribute to an increased risk of cerebral aneurysm pathogenesis and rupture in menopausal and postmenopausal women. Med Hypotheses. 2006;66(4):736-56.

15 Longstreth WT, Nelson LM, Koepsell TD, van Belle G. Subarachnoid hemorrhage and hormonal factors in women. A population-based case-control study. Ann Intern Med. 1994 Aug;121(3):168-73.

16 Hademenos GJ, Massoud TF, Turjman F, Sayre JW. Anatomical and morphological factors correlating with rupture of intracranial aneurysms in patients referred for endovascular treatment. Neuroradiology. 1998 Nov;40(11):755-60. 